\title{
New Explicit Bounds on Gamidov Type Integral Inequalities for Functions in Two Variables and Their Applications
}

\author{
Kelong Cheng' and Chunxiang Guo ${ }^{2}$ \\ ${ }^{1}$ School of Science, Southwest University of Science and Technology, Mianyang 621010, China \\ ${ }^{2}$ School of Business, Sichuan University, Chengdu 610064, China \\ Correspondence should be addressed to Chunxiang Guo; guocx70@163.com
}

Received 14 February 2014; Accepted 10 June 2014; Published 22 June 2014

Academic Editor: Praveen Agarwal

Copyright (c) $2014 \mathrm{~K}$. Cheng and C. Guo. This is an open access article distributed under the Creative Commons Attribution License, which permits unrestricted use, distribution, and reproduction in any medium, provided the original work is properly cited.

Some linear and nonlinear Gamidov type integral inequalities in two variables are established, which can give the explicit bounds on the solutions to a class of Volterra-Fredholm integral equations. Some examples of application are presented to show boundedness and uniqueness of solutions of a Volterra-Fredholm type integral equation.

\section{Introduction}

Integral inequalities that give explicit bounds on unknown functions provide a very useful and important device in the study of many qualitative as well as quantitative properties of solutions of differential and integral equations. During the past few years, an enormous amount of effort has been devoted to the discovery of new types of inequalities and their applications in various branches of ordinary and partial differential and integral equations; see [1-14] and the references given therein.

In particular, Gamidov [6], while studying the boundary value problem for higher order differential equations, initiated the study of obtaining explicit upper bounds on the integral inequalities of the forms

$$
u(t) \leq c+\int_{\alpha}^{t} f(s) u(s) d s+\int_{\alpha}^{\beta} g(s) u(s) d s
$$

for $t \in[\alpha, \beta]$, under some suitable conditions on the functions involved in (1). Later, such inequalities (usually called Gamidov type inequalities) are also discussed by Bainov and Simeonov [15] and Pachpatte [16, 17]. In [16],
Pachpatte established more general Gamidov inequalities as follows:

$$
u(t) \leq c+\int_{\alpha}^{t} a(t, s) u(s) d s+\int_{\alpha}^{\beta} b(t, s) u(s) d s
$$

and also generalized it to a general version in two independent variables [18] as follows:

$$
\begin{aligned}
u(x, y) \leq & c+\int_{x_{0}}^{x} \int_{y_{0}}^{y} a(x, y, s, t) u(s) d s d t \\
& +\int_{x_{0}}^{M} \int_{y_{0}}^{N} b(x, y, s, t) u(s) d s d t .
\end{aligned}
$$

Based on the results of inequalities (3), the retarded cases with power nonlinearity of inequalities (3) are studied by Ma and Pečarić [19]. As a matter of fact, the results of inequality (2) are not right. In the proof of Theorem 1 in [16] about inequalities (2), by definition $z(\alpha)=c+\int_{\alpha}^{\beta} b(t, s) u(s) d s$ and inequalities $u(t) \leq z(\alpha) \exp \left(\int_{\alpha}^{t} a(t, \sigma) d \sigma\right)$, they obtained $z(\alpha) \leq c /(1-p(t))$ (for details, see [16, page 355]). However, we can see easily that $z(\alpha)$ is a function in $t$, while it was treated as a constant in [16].

In this paper, we discuss some linear and nonlinear Gamidov type integral inequalities and obtain new explicit bounds on these inequalities. Moreover, we also give some 
examples to show boundedness and uniqueness of solutions of a Volterra-Fredholm type integral equation.

\section{Linear Gamidov Type Integral Inequalities}

In what follows, $R$ denotes the set of real numbers, $R_{+}=$ $[0, \infty), R_{0}=(0, \infty), I_{1}=[0, M]$, and $I_{2}=[0, N]$ are given subsets of $R$. Let $\Delta=I_{1} \times I_{2} . C(U, V)$ denotes the collection of continuous functions from $U$ to $V$.

Lemma 1. Assume $u(x, y), a(x, y), c(x, y), g(x, y) \in$ $C\left(\Delta, R_{+}\right)$and

$$
u(x, y) \leq a(x, y)+c(x, y) \int_{0}^{M} \int_{0}^{N} g(s, t) u(s, t) d s d t
$$

for $(x, y) \in \Delta$. If $\int_{0}^{M} \int_{0}^{N} c(s, t) g(s, t) d s d t<1$, then the following explicit estimate

$$
u(x, y) \leq a(x, y)+\frac{c(x, y) \int_{0}^{M} \int_{0}^{N} a(s, t) g(s, t) d s d t}{1-\int_{0}^{M} \int_{0}^{N} c(s, t) g(s, t) d s d t}
$$

holds for $(x, y) \in \Delta$.

Proof. Obviously, $\int_{0}^{M} \int_{0}^{N} g(s, t) u(s, t) d s d t$ is a constant. Letting $\int_{0}^{M} \int_{0}^{N} g(s, t) u(s, t) d s d t=k$, from (4), we have

$$
u(x, y) \leq a(x, y)+c(x, y) k .
$$

Since $g(x, y)$ is positive, then

$$
g(x, y) u(x, y) \leq a(x, y) g(x, y)+c(x, y) g(x, y) k .
$$

Integrating for both sides of (7) on $\Delta$, we have

$$
\begin{aligned}
k= & \int_{0}^{M} \int_{0}^{N} g(s, t) u(s, t) d s d t \\
\leq & \int_{0}^{M} \int_{0}^{N} a(s, t) g(s, t) d s d t \\
& +k \int_{0}^{M} \int_{0}^{N} c(s, t) g(s, t) d s d t .
\end{aligned}
$$

Consequently, it follows from the inequality above that

$$
k \leq \frac{\int_{0}^{M} \int_{0}^{N} a(s, t) g(s, t) d s d t}{1-\int_{0}^{M} \int_{0}^{N} c(s, t) g(s, t) d s d t}
$$

where $\int_{0}^{M} \int_{0}^{N} c(s, t) g(s, t) d s d t<1$. Substituting the inequality above into (6), we get the explicit estimate (5) for $u(x, y)$.

Theorem 2. Assume $a(x, y), b(x, y), c(x, y), f(x, y)$, $g(x, y) \in C\left(\Delta, R_{+}\right)$, and $a(x, y), b(x, y), c(x, y)$ are nondecreasing in $x$ and $y$. If $u(x, y) \in C\left(\Delta, R_{+}\right)$satisfies

$$
\begin{aligned}
u(x, y) \leq & a(x, y)+b(x, y) \int_{0}^{x} \int_{0}^{y} f(s, t) u(s, t) d s d t \\
& +c(x, y) \int_{0}^{M} \int_{0}^{N} g(s, t) u(s, t) d s d t,
\end{aligned}
$$

then, under the condition that

$$
\begin{aligned}
& \int_{0}^{M} \int_{0}^{N} c(s, t) g(s, t) \exp \left\{b(s, t) \int_{0}^{s} \int_{0}^{t} f(u, v) d u d v\right\} d s d t \\
& <1
\end{aligned}
$$

the following explicit estimate

$$
\begin{aligned}
& u(x, y) \\
& \leq[a(x, y)+c(x, y) \\
& \times\left(\left(\int_{0}^{M} \int_{0}^{N} a(s, t) g(s, t)\right.\right. \\
& \times\left(1-\int_{0}^{M} \int_{0}^{N} c(s, t) g(s, t)\right. \\
& \left.\times \exp \left\{b(s) \int_{0}^{s} \int_{0}^{t} f(u, v) d u d v\right\} d s d t\right) \\
& \times \exp \left\{b(x, y) \int_{0}^{x} \int_{0}^{y} f(s, t) d s d t\right\}
\end{aligned}
$$

holds for $(x, y) \in \Delta$.

Proof. Fixing any arbitrary $(X, Y) \in \Delta$, then for $(x, y) \in \Delta_{1}=$ $[0, X] \times[0, Y]$, from $(10)$, we have

$$
\begin{aligned}
u(x, y) \leq & a(X, Y)+b(X, Y) \int_{0}^{x} \int_{0}^{y} f(s, t) u(s, t) d s d t \\
& +c(X, Y) \int_{0}^{M} \int_{0}^{N} g(s, t) u(s, t) d s d t
\end{aligned}
$$

where we apply that $a(x, y), b(x, y)$, and $c(x, y)$ are nondecreasing in $x$ and $y$.

Define a function $v(x, y),(x, y) \in \Delta_{1}$ by the right side of (13). Then, $v(x, y)$ is positive and nondecreasing in $x$ and $y$, and

$$
u(x, y) \leq v(x, y)
$$


Furthermore, we have

$$
\begin{aligned}
v(0, y)=a(X, Y) & +c(X, Y) \int_{0}^{M} \int_{0}^{N} g(s, t) u(s, t) d s d t \\
\frac{\partial}{\partial x} v(x, y) & =b(X, Y) \int_{0}^{y} f(x, t) u(x, t) d t \\
& \leq b(X, Y) \int_{0}^{y} f(x, t) v(x, t) d t \\
& \leq v(x, y) b(X, Y) \int_{0}^{y} f(x, t) d t
\end{aligned}
$$

Since $v(x, y)$ is nondecreasing in $y$, from (16), one gets

$$
\frac{(\partial / \partial x) v(x, y)}{v(x, y)} \leq b(X, Y) \int_{0}^{y} f(x, t) d t
$$

Keeping $y$ fixed in (17), setting $x=s$, and integrating it from 0 to $x$, we get

$$
v(x, y) \leq v(0, y) \exp \left\{b(X, Y) \int_{0}^{x} \int_{0}^{y} f(s, t) d s d t\right\} .
$$

It follows from (14) and (15) that

$$
\begin{aligned}
& u(x, y) \\
& \leq[a(X, Y) \\
& \left.\quad+c(X, Y) \int_{0}^{M} \int_{0}^{N} g(s, t) u(s, t) d s d t\right] \\
& \quad \times \exp \left\{b(X, Y) \int_{0}^{x} \int_{0}^{y} f(s, t) d s d t\right\} \\
& =a(X, Y) \exp \left\{b(X, Y) \int_{0}^{x} \int_{0}^{y} f(s, t) d s d t\right\} \\
& +c(X, Y) \exp \left\{b(X, Y) \int_{0}^{x} \int_{0}^{y} f(s, t) d s d t\right\} \\
& \quad \times \int_{0}^{M} \int_{0}^{N} g(s, t) u(s, t) d s d t \\
& =\widetilde{A}(x, y ; X, Y) \\
& +\widetilde{C}(x, y ; X, Y) \int_{0}^{M} \int_{0}^{N} g(s, t) u(s, t) d s d t
\end{aligned}
$$

where

$$
\begin{aligned}
& \widetilde{A}(x, y ; X, Y)=a(X, Y) \exp \left\{b(X, Y) \int_{0}^{x} \int_{0}^{y} f(s, t) d s d t\right\}, \\
& \widetilde{C}(x, y ; X, Y)=c(X, Y) \exp \left\{b(X, Y) \int_{0}^{x} \int_{0}^{y} f(s, t) d s d t\right\} .
\end{aligned}
$$

Now, a suitable application of Lemma 1 yields

$$
\begin{aligned}
& u(x, y) \\
& \leq \widetilde{A}(x, y ; X, Y)+\widetilde{C}(x, y ; X, Y) \\
& \quad \times \frac{\int_{0}^{M} \int_{0}^{N} \widetilde{A}(s, t ; X, Y) g(s, t) d s d t}{1-\int_{0}^{M} \int_{0}^{N} \widetilde{C}(s, t ; X, Y) g(s, t) d s d t} .
\end{aligned}
$$

Since the inequality above holds for all $(x, y) \in \Delta_{1}$, taking $x=X$ and $y=Y$ in (21), we have

$$
\begin{aligned}
u(X, Y) \leq & \widetilde{A}(X, Y ; X, Y)+\widetilde{C}(X, Y ; X, Y) \\
& \times \frac{\int_{0}^{M} \int_{0}^{N} \widetilde{A}(s, t ; X, Y) g(s, t) d s d t}{1-\int_{0}^{M} \int_{0}^{N} \widetilde{C}(s, t ; X, Y) g(s, t) d s d t} \\
= & \widehat{A}(X, Y)+\widehat{C}(X, Y) \\
& \times \frac{\int_{0}^{M} \int_{0}^{N} \widehat{A}(s, t) g(s, t) d s d t}{1-\int_{0}^{M} \int_{0}^{N} \widehat{C}(s, t) g(s, t) d s d t},
\end{aligned}
$$

for $(X, Y) \in \Delta$, where

$$
\begin{aligned}
& \widehat{A}(X, Y)=a(X, Y) \exp \left\{b(X, Y) \int_{0}^{X} \int_{0}^{Y} f(s, t) d s d t\right\}, \\
& \widehat{C}(X, Y)=c(X, Y) \exp \left\{b(X, Y) \int_{0}^{X} \int_{0}^{Y} f(s, t) d s d t\right\} .
\end{aligned}
$$

Substituting (23) into (22) and simplifying it, we get

$$
\begin{aligned}
& u(X, Y) \\
& \leq[a(X, Y)+c(X, Y) \\
& \times\left(\left(\int_{0}^{M} \int_{0}^{N} a(s, t) g(s, t)\right.\right. \\
& \times\left(1-\int_{0}^{M} \int_{0}^{N} c(s, t) g(s, t)\right. \\
& \left.\times \exp \left\{b(s, t) \int_{0}^{s} \int_{0}^{t} f(u, v) d u d v\right\} d s d t\right) \\
& \times \exp \left\{b(s, t) \int_{0}^{s} \int_{0}^{t} f(u, v) d u d v\right\}
\end{aligned}
$$$$
\times \exp \left\{b(X, Y) \int_{0}^{X} \int_{0}^{Y} f(s, t) d s d t\right\} .
$$

Considering that $X$ and $Y$ are arbitrary, we replace $X$ and $Y$ by $x$ and $y$, respectively, and get the desired estimate (12). The proof is complete. 
Remark 3. If $a(x, y), b(x, y)$, and $c(x, y)$ are not nondecreasing, our result also holds, since we can replace it by $\widetilde{a}(x, y)=$ $\max _{0 \leq s \leq x, 0 \leq t \leq y} a(s, t), \tilde{b}(x, y)=\max _{0 \leq s \leq x, 0 \leq t \leq y} b(s, t)$, and $\widetilde{c}(x, y)=\max _{0 \leq s \leq x, 0 \leq t \leq y} c(s, t)$.

Remark 4. As for inequality (3) discussed by Pachpatte [18] or a more general case as follows:

$$
\begin{aligned}
u(x, y) \leq & a(x, y)+\int_{0}^{x} \int_{0}^{y} \tilde{f}(x, y, s, t) u(s) d s d t \\
& +\int_{0}^{M} \int_{0}^{N} \tilde{g}(x, y, s, t) u(s) d s d t
\end{aligned}
$$

there are some technical problems which we do not discuss here. Clearly, inequalities (4) are the special case of inequalities (25); that is, $\widetilde{f}(x, y, s, t)=b(x, y) f(s, t)$ and $\widetilde{g}(x, y, s, t)=$ $c(x, y) g(s, t)$.

\section{Nonlinear Gamidov Type Integral Inequalities}

In this section, we discuss some integral inequalities with some power nonlinear terms.

Lemma 5 (see [20]). Let $a \geq 0, m \geq n \geq 0$, and $m \neq 0$. Then

$$
a^{n / m} \leq \frac{n}{m} K^{(n-m) / m} a+\frac{m-n}{m} K^{n / m}
$$

for any $K>0$.

Theorem 6. Assume that $a(x, y), b(x, y), c(x, y), f(x, y)$, and $g(x, y)$ are defined as in Theorem 2. If $u(x, y) \in C\left(\Delta, R_{+}\right)$ satisfies

$$
\begin{aligned}
u^{m}(x, y) \leq & a(x, y)+b(x, y) \int_{0}^{x} \int_{0}^{y} f(s, t) u^{n}(s, t) d s d t \\
& +c(x, y) \int_{0}^{M} \int_{0}^{N} g(s, t) u^{r}(s, t) d s d t
\end{aligned}
$$

where $m \geq n \geq 0, m \geq r \geq 0$, and $m$, $n$, and $r$ are constants, then, under the condition that

$$
\begin{aligned}
& \int_{0}^{M} \int_{0}^{N} \bar{C}(s, t) \bar{G}(s, t) \exp \left\{\bar{B}(s, t) \int_{0}^{s} \int_{0}^{t} \bar{F}(u, v) d u d v\right\} d s d t \\
& \quad<1,
\end{aligned}
$$

the following explicit estimate

$$
\begin{aligned}
& u(x, y) \\
& \leq\{a(x, y) \\
& +[\bar{A}(x, y)+\bar{C}(x, y) \\
& \times\left(\int_{0}^{M} \int_{0}^{N} \bar{A}(s, t) \bar{G}(s, t)\right. \\
& \times \exp \{\bar{B}(s, t) \\
& \times\left(1-\int_{0}^{M} \int_{0}^{N} \bar{C}(s, t) \bar{G}(s, t)\right. \\
& \left.\left.\left.\times \exp \left\{\bar{B}(x, y) \int_{0}^{x} \int_{0}^{y} \bar{F}(s, t) d s d t\right\}\right\}_{0}^{t} \bar{F}(u, v) d u d v\right\} d s d t\right) \\
& \times \exp \left\{\bar{B}(s, t) \int_{0}^{s} \int_{0}^{t} \bar{F}(u, v) d u d v\right\} \\
& \left.\left.\times d s d t)^{-1}\right)\right]
\end{aligned}
$$

holds for $(x, y) \in \Delta$, where

$$
\begin{aligned}
& \bar{A}(x, y) \\
& =b(x, y) \int_{0}^{x} \int_{0}^{y} f(s, t) \\
& \times\left[\frac{n}{m} K_{1}^{(n-m) / m}(s, t) a(s, t)\right. \\
& \left.+\frac{m-n}{m} K_{1}^{n / m}(s, t)\right] d s d t \\
& +c(x, y) \int_{0}^{M} \int_{0}^{N} g(s, t) \\
& \bar{B}(x, y)=\frac{n}{m} b(x, y), \quad \bar{C}(x, y)=\frac{n}{m} c(x, y), \\
& \bar{F}(x, y)=f(x, y) K_{1}^{(n-m) / m}(x, y), \\
& \bar{G}(x, y)=g(x, y) K_{2}^{(r-m) / m}(x, y)
\end{aligned}
$$

for any $K_{i}(x, y) \in C\left(\Delta, R_{0}\right)(i=1,2)$. 
Proof. Define a function $w(x, y)$ by

$$
\begin{aligned}
w(x, y)= & b(x, y) \int_{0}^{x} \int_{0}^{y} f(s, t) u^{n}(s, t) d s d t \\
& +c(x, y) \int_{0}^{M} \int_{0}^{N} g(s, t) u^{r}(s, t) d s d t
\end{aligned}
$$

for $(x, y) \in \Delta$. Then, from (27), we have

$$
u^{m}(x, y) \leq a(x, y)+w(x, y)
$$

or

$$
u(x, y) \leq(a(x, y)+w(x, y))^{1 / m}
$$

Applying Lemma 5, for any $K_{i}(x, y) \in C\left(\Delta, R_{0}\right)(i=1,2)$, we get

$$
\begin{aligned}
u^{n}(x, y) \leq & (a(x, y)+w(x, y))^{n / m} \\
\leq & \frac{n}{m} K_{1}^{(n-m) / m}(x, y)(a(x, y)+w(x, y)) \\
& +\frac{m-n}{m} K_{1}^{n / m}(x, y), \\
u^{r}(x, y) \leq & (a(x, y)+w(x, y))^{r / m} \\
\leq & \frac{r}{m} K_{2}^{(r-m) / m}(x, y)(a(x, y)+w(x, y)) \\
& +\frac{m-r}{m} K_{2}^{r / m}(x, y) .
\end{aligned}
$$

Substituting (34) into (31), we get

$$
\begin{aligned}
& w(x, y) \\
& \leq b(x, y) \int_{0}^{x} \int_{0}^{y} f(s, t) \\
& \times\left[\frac{n}{m} K_{1}^{(n-m) / m}(s, t)(a(s, t)+w(s, t))\right. \\
& \left.+\frac{m-n}{m} K_{1}^{n / m}(s, t)\right] d s d t \\
& +c(x, y) \int_{0}^{M} \int_{0}^{N} g(s, t) \\
& \times\left[\frac{r}{m} K_{2}^{(r-m) / m}(s, t)(a(s, t)+w(s, t))\right. \\
& \left.+\frac{m-r}{m} K_{2}^{r / m}(s, t)\right] d s d t \\
& =b(x, y) \int_{0}^{x} \int_{0}^{y} f(s, t)\left[\frac{n}{m} K_{1}^{(n-m) / m}(s, t) a(s, t)\right. \\
& \left.+\frac{m-n}{m} K_{1}^{n / m}(s, t)\right] d s d t
\end{aligned}
$$

$$
\begin{aligned}
& +c(x, y) \int_{0}^{M} \int_{0}^{N} g(s, t)\left[\frac{r}{m} K_{2}^{(r-m) / m}(s, t) a(s, t)\right. \\
& \left.+\frac{m-r}{m} K_{2}^{r / m}(s, t)\right] d s d t \\
& +\frac{n}{m} b(x, y) \int_{0}^{x} \int_{0}^{y} f(s, t) K_{1}^{(n-m) / m}(s, t) w(s, t) d s d t \\
& +\frac{r}{m} c(x, y) \int_{0}^{M} \int_{0}^{N} g(s, t) K_{2}^{(r-m) / m}(s, t) w(s, t) d s d t \\
& =\bar{A}(x, y)+\bar{B}(x, y) \int_{0}^{x} \int_{0}^{y} \bar{F}(s, t) w(s, t) d s d t \\
& +\bar{C}(x, y) \int_{0}^{M} \int_{0}^{N} \bar{G}(s, t) w(s, t) d s d t
\end{aligned}
$$

which is similar to inequality (10), where $\bar{A}(x, y), \bar{B}(x, y)$, $\bar{C}(x, y), \bar{F}(x, y)$, and $\bar{G}(x, y)$ are defined as in (30). It is easy to see that $\bar{A}(x, y), \bar{B}(x, y)$, and $\bar{C}(x, y)$ are nonnegative, continuous, and nondecreasing for $(x, y) \in \Delta$.

A suitable application of Theorem 2 to (35) yields

$$
\begin{aligned}
& w(x, y) \\
& \leq[\bar{A}(x, y)+\bar{C}(x, y)
\end{aligned}
$$

$$
\begin{aligned}
& \times\left(\int_{0}^{M} \int_{0}^{N} \bar{A}(s, t) \bar{G}(s, t)\right. \\
& \left.\times \exp \left\{\bar{B}(s, t) \int_{0}^{s} \int_{0}^{t} \bar{F}(u, v) d u d v\right\} d s d t\right) \\
& \times\left(1-\int_{0}^{M} \int_{0}^{N} \bar{C}(s, t) \bar{G}(s, t)\right. \\
& \times \exp \left\{\bar{B}(s, t) \int_{0}^{s} \int_{0}^{t} \bar{F}(u, v) d u d v\right\} \\
& \left.\left.\left.\times d s d t)^{-1}\right)\right]_{0}^{x} \int_{0}^{y} \bar{F}(s, t) d s d t\right\} .
\end{aligned}
$$

Substituting (36) into (33), we can get (29). 
Remark 7. In [19], Ma and Pečarić discussed the retarded Gamidov type integral inequality:

$$
\begin{aligned}
u^{m}(x, y) \leq & a(x, y)+\int_{0}^{\alpha(x)} \int_{0}^{\beta(y)} f(x, y, s, t) u^{n}(s, t) d s d t \\
& +\int_{0}^{\alpha(M)} \int_{0}^{\beta(N)} g(x, y, s, t) u^{r}(s, t) d s d t
\end{aligned}
$$

As mentioned above, the results of inequality (3) obtained by Pachpatte [18] are not right. So the results in [19] are not valid.

When $m=2, n=r=1$ in Theorem 6, an Ou-Iang version of Gamidov type inequality is obtained as follows.

Corollary 8. Assume that $a(x, y), b(x, y), c(x, y), f(x, y)$, and $g(x, y)$ are defined as in Theorem 2. If $u(x, y) \in C\left(\Delta, R_{+}\right)$ satisfies

$$
\begin{aligned}
u^{2}(x, y) \leq & a(x, y)+b(x, y) \int_{0}^{x} \int_{0}^{y} f(s, t) u(s, t) d s d t \\
& +c(x, y) \int_{0}^{M} \int_{0}^{N} g(s, t) u(s, t) d s d t
\end{aligned}
$$

Then, under the condition that

$$
\begin{aligned}
& \int_{0}^{M} \int_{0}^{N} \frac{1}{2} c(s, t) \bar{G}(s, t) \\
& \quad \times \exp \left\{\frac{1}{2} b(s, t) \int_{0}^{s} \int_{0}^{t} \bar{F}(u, v) d u d v\right\} d s d t<1,
\end{aligned}
$$

the following explicit estimate

$$
\begin{aligned}
& u(x, y) \\
& \leq\{a(x, y) \\
& +\left[\bar{A}(x, y)+\frac{1}{2} c(x, y)\right. \\
& \times\left(\left(\int_{0}^{M} \int_{0}^{N} \bar{A}(s, t) \bar{G}(s, t)\right.\right. \\
& \quad \times \exp \left\{\frac{1}{2} b(s, t) \int_{0}^{s} \int_{0}^{t} \bar{F}(u, v) d u d v\right\} \\
& \times d s d t)
\end{aligned}
$$

$$
\begin{gathered}
\times\left(1-\int_{0}^{M} \int_{0}^{N} \frac{1}{2} c(s, t) \bar{G}(s, t)\right. \\
\quad \times \exp \left\{\frac{1}{2} b(s, t) \int_{0}^{s} \int_{0}^{t} \bar{F}(u, v) d u d v\right\} \\
\left.\left.\times d s d t)^{-1}\right)\right] \\
\left.\times \exp \left\{\frac{1}{2} b(x, y) \int_{0}^{x} \int_{0}^{y} \bar{F}(s, t) d s d t\right\}\right\}^{1 / 2},
\end{gathered}
$$

holds for $(x, y) \in \Delta$, where

$\bar{A}(x, y)$

$$
\begin{aligned}
&=b(x, y) \int_{0}^{x} \int_{0}^{y} \frac{1}{2} f(s, t) \\
& \times\left[K_{1}^{-1 / 2}(s, t) a(s, t)+K_{1}^{1 / 2}(s, t)\right] d s d t \\
&+c(x, y) \int_{0}^{M} \int_{0}^{N} \frac{1}{2} g(s, t) \\
& \times\left[K_{2}^{-1 / 2}(s, t) a(s, t)+K_{2}^{1 / 2}(s, t)\right] d s d t
\end{aligned}
$$

$\bar{F}(x, y)=f(x, y) K_{1}^{-1 / 2}(x, y)$,

$\bar{G}(x, y)=g(x, y) K_{2}^{-1 / 2}(x, y)$

for any $K_{i}(x, y) \in C\left(\Delta, R_{0}\right)(i=1,2)$.

When $m=n=1,0 \leq r \leq 1$ in Theorem 6 , we can get an interesting result as follows.

Corollary 9. Assume that $a(x, y), b(x, y), c(x, y), f(x, y)$, and $g(x, y)$ are defined as in Theorem 2. If $u(x, y) \in C\left(\Delta, R_{+}\right)$ satisfies

$$
\begin{aligned}
u(x, y) \leq & a(x, y)+b(x, y) \int_{0}^{x} \int_{0}^{y} f(s, t) u(s, t) d s d t \\
& +c(x, y) \int_{0}^{M} \int_{0}^{N} g(s, t) u^{r}(s, t) d s d t,
\end{aligned}
$$

then, under the condition that

$$
\begin{aligned}
& \int_{0}^{M} \int_{0}^{N} r c(s, t) \bar{G}(s, t) \\
& \quad \times \exp \left\{b(s, t) \int_{0}^{s} \int_{0}^{t} \bar{F}(u, v) d u d v\right\} d s d t<1,
\end{aligned}
$$


the following explicit estimate

$$
\begin{aligned}
& u(x, y) \\
& \leq\{a(x, y) \\
& +[\bar{A}(x, y)+c(x, y) \\
& \times\left(\left(\int_{0}^{M} \int_{0}^{N} \bar{A}(s, t) \bar{G}(s, t)\right.\right. \\
& \left.\times \exp \left\{b(x, y) \int_{0}^{x} \int_{0}^{y} \bar{F}(s, t) d s d t\right\}\right\}_{0}^{1 / 2}, \\
& \times\left(1-\int_{0}^{M} \int_{0}^{N} r c(s, t) \bar{G}(s, t)\right. \\
& \left.\times d s d t) \int_{0}^{t} \bar{F}(u, v) d u d v\right\} \\
& \times \exp \left\{b(s, t) \int_{0}^{s} \int_{0}^{t} \bar{F}(u, v) d u d v\right\}
\end{aligned}
$$

holds for $(x, y) \in \Delta$, where

$$
\begin{aligned}
& \bar{A}(x, y) \\
& =b(x, y) \int_{0}^{x} \int_{0}^{y} f(s, t) a(s, t) d s d t \\
& +c(x, y) \int_{0}^{M} \int_{0}^{N} g(s, t) \\
& \times\left[r K^{r-1}(s, t) a(s, t)\right. \\
& \left.\quad+(1-r) K^{r}(s, t)\right] d s d t, \\
& \bar{G}(x, y)=g(x, y) K^{r-1}(x, y)
\end{aligned}
$$

for any $K(x, y) \in C\left(\Delta, R_{0}\right)$.

\section{Applications}

In this section, we present some applications of our results to study boundedness and uniqueness of solutions of a VolterraFredholm integral equation in two independent variables.
Considering the following integral equation:

$$
\begin{aligned}
z^{m}(x, y)= & a(x, y)+b(x, y) \int_{0}^{x} \int_{0}^{y} F(s, t, z) d s d t \\
& +c(x, y) \int_{0}^{M} \int_{0}^{N} G(s, t, z) d s d t,
\end{aligned}
$$

for $(x, y) \in \Delta$, where $z(x, y) \in C(\Delta, R), a(x, y), b(x, y)$, $c(x, y) \in C\left(\Delta, R_{+}\right), F(x, y, z), G(x, y, z) \in C(\Delta \times R, R)$, and $m>1$ is a constant.

Theorem 10. Assume that the functions $F$ and $G$ in (46) satisfy the conditions

$$
\begin{aligned}
& |F(s, t, z)| \leq f(s, t)|z|^{n}, \\
& |G(s, t, z)| \leq g(s, t)|z|^{r},
\end{aligned}
$$

where $f(s, t)$ and $g(s, t)$ are defined as in Theorem 2, $n$ and $r$ satisfying $0<n \leq m, 0<r \leq m$ are constants. If

$$
\begin{aligned}
& \int_{0}^{M} \int_{0}^{N} \bar{C}(s, t) \bar{G}(s, t) \exp \left\{\bar{B}(s, t) \int_{0}^{s} \int_{0}^{t} \bar{F}(u, v) d u d v\right\} d s d t \\
& \quad<1
\end{aligned}
$$

and $z(x, y)$ is a solution of (46) and (47), then

$$
\begin{aligned}
& \quad|z(x, y)| \\
& \leq\{a(x, y) \\
& \quad+[\bar{A}(x, y)+\bar{C}(x, y)
\end{aligned}
$$$$
\times\left(\left(\int_{0}^{M} \int_{0}^{N} \bar{A}(s, t) \bar{G}(s, t)\right.\right.
$$$$
\times \exp \left\{\bar{B}(s, t) \int_{0}^{s} \int_{0}^{t} \bar{F}(u, v) d u d v\right\}
$$$$
\times d s d t)
$$$$
\times\left(1-\int_{0}^{M} \int_{0}^{N} \bar{C}(s, t) \bar{G}(s, t)\right.
$$$$
\times \exp \left\{\bar{B}(s, t) \int_{0}^{s} \int_{0}^{t} \bar{F}(u, v) d u d v\right\}
$$$$
\left.\left.\times d s d t)^{-1}\right)\right]
$$

$$
\left.\times \exp \left\{\bar{B}(x, y) \int_{0}^{x} \int_{0}^{y} \bar{F}(s, t) d s d t\right\}\right\}^{1 / m},
$$

for $(x, y) \in \Delta$ and any $K_{i}(x, y) \in C\left(\Delta, R_{0}\right)(i=1,2)$, where $\bar{A}(x, y), \bar{B}(x, y), \bar{C}(x, y), \bar{F}(x, y)$, and $\bar{G}(x, y)$ are defined as in Theorem 6. 
Proof. Letting $z(x, y)$ be a solution of (46), from (47), we have

$$
\begin{aligned}
|z(x, y)|^{m} \leq & |a(x, y)| \\
& +b(x, y) \int_{0}^{x} \int_{0}^{y} f(s, t)|z(s, t)|^{n} d s d t \\
& +c(x, y) \int_{0}^{M} \int_{0}^{N} g(s, t)|z(s, t)|^{r} d s d t .
\end{aligned}
$$

An application of the inequality given in Theorem 6 to (50) yields (49). The right-hand side of (49) gives us the bound on the solution $z(x, y)$ of (46) in terms of the unknown functions.

Considering the following integral equation:

$$
\begin{aligned}
z(x, y)= & a(x, y)+b(x, y) \int_{0}^{x} \int_{0}^{y} F(s, t, z) d s d t \\
& +c(x, y) \int_{0}^{M} \int_{0}^{N} G(s, t, z) d s d t,
\end{aligned}
$$

for $(x, y) \in \Delta$, where $z(x, y) \in C(\Delta, R), a(x, y), b(x, y)$, $c(x, y) \in C\left(\Delta, R_{+}\right), F(x, y, z), G(x, y, z) \in C(\Delta \times R, R)$.

Theorem 11. Assume that the functions $F$ and $G$ in (46) satisfy the conditions

$$
\begin{aligned}
& |F(s, t, z)-F(s, t, \widetilde{z})| \leq f(s, t)|z-\widetilde{z}|, \\
& |G(s, t, z)-G(s, t, \widetilde{z})| \leq g(s, t)|z-\widetilde{z}|^{r},
\end{aligned}
$$

where $f(s, t)$ and $g(s, t)$ are defined as in Theorem $2,0<r \leq 1$ is a constant. If

$$
\begin{array}{rl}
\int_{0}^{M} \int_{0}^{N} & r c(s, t) \bar{G}(s, t) \\
& \times \exp \left\{b(s, t) \int_{0}^{s} \int_{0}^{t} \bar{F}(u, v) d u d v\right\} d s d t<1,
\end{array}
$$

where $\bar{F}$ and $\bar{G}$ are defined as in Corollary 9 and $z(x, y)$ is a solution of (51) and (52), then (51) has at most one solution.

Proof. Let $z(x, y)$ and $\widetilde{z}(x, y)$ be two solutions of (51); that is,

$$
\begin{aligned}
\widetilde{z}(x, y)= & a(x, y)+b(x, y) \int_{0}^{x} \int_{0}^{y} F(s, t, \widetilde{z}) d s d t \\
& +c(x, y) \int_{0}^{M} \int_{0}^{N} G(s, t, \widetilde{z}) d s d t .
\end{aligned}
$$

From (51) and (54), we have

$$
\begin{aligned}
& |z(x, y)-\tilde{z}(x, y)| \\
& \leq b(x, y) \int_{0}^{x} \int_{0}^{y}|F(s, t, z)-F(s, t, \widetilde{z})| d s d t \\
& \quad+c(x, y) \int_{0}^{M} \int_{0}^{N}|G(s, t, z)-G(s, t, \tilde{z})| d s d t \\
& \leq b(x, y) \int_{0}^{x} \int_{0}^{y} f(s, t)|z-\widetilde{z}| d s d t \\
& \quad+c(x, y) \int_{0}^{M} \int_{0}^{N} g(s, t)|z-\widetilde{z}|^{r} d s d t .
\end{aligned}
$$

According to Corollary 9, we obtain that $|z(x, y)-\widetilde{z}(x, y)| \leq$ 0 , which implies $z(x, y)=\widetilde{z}(x, y)$ for $(x, y) \in \Delta$.

\section{Conflict of Interests}

The authors declare that there is no conflict of interests regarding the publication of this paper.

\section{Acknowledgments}

The authors are very grateful to the referees for their helpful comments and valuable suggestions. This work is supported by the Doctoral Program Research Funds of Southwest University of Science and Technology (no. 11zx7129) and the Fundamental Research Funds for the Central Universities (no. skqy201324).

\section{References}

[1] W.-S. Cheung and Q.-H. Ma, "On certain new GronwallOu-Iang type integral inequalities in two variables and their applications," Journal of Inequalities and Applications, no. 4, pp. 347-361, 2005.

[2] S. Deng and C. Prather, "Generalization of an impulsive nonlinear singular Gronwall-Bihari inequality with delay," Journal of Inequalities in Pure and Applied Mathematics, vol. 9, no. 2, article 34, 2008.

[3] S. Deng, "Nonlinear discrete inequalities with two variables and their applications," Applied Mathematics and Computation, vol. 217, no. 5, pp. 2217-2225, 2010.

[4] S. S. Dragomir and Y.-H. Kim, "Some integral inequalities for functions of two variables," Electronic Journal of Differential Equations, vol. 2003, article 10, 2003.

[5] S. S. Dragomir, Some Gronwall type inequalities and Applications, Nova Science Publishers, New York, NY, USA, 2003.

[6] S. G. Gamidov, "Integral inequalities for boundary value problems for differential equations," Differential Equations, vol. 5, pp. 463-472, 1969.

[7] Y.-H. Kim, "Gronwall, Bellman and Pachpatte type integral inequalities with applications," Nonlinear Analysis: Theory, Methods \& Applications, vol. 71, no. 12, pp. e2641-e2656, 2009.

[8] M. Medved', "Nonlinear singular integral inequalities for functions in two and $n$ independent variables," Journal of Inequalities and Applications, vol. 5, no. 3, pp. 287-308, 2000.

[9] Y. Tian, M. Fan, and F. Meng, "A generalization of retarded integral inequalities in two independent variables and their applications," Applied Mathematics and Computation, vol. 221, pp. 239-248, 2013.

[10] W.-S. Wang, "A generalized retarded Gronwall-like inequality in two variables and applications to BVP," Applied Mathematics and Computation, vol. 191, no. 1, pp. 144-154, 2007.

[11] W.-S. Wang and C.-X. Shen, "On a generalized retarded integral inequality with two variables," Journal of Inequalities and Applications, vol. 2008, Article ID 518646, 2008.

[12] H. Zhang and F. Meng, "On certain integral inequalities in two independent variables for retarded equations," Applied Mathematics and Computation, vol. 203, no. 2, pp. 608-616, 2008.

[13] K. Zheng, "Some retarded nonlinear integral inequalities in two variables and applications," Journal of Inequalities in Pure and Applied Mathematics, vol. 9, no. 2, article 57, 2008. 
[14] K. Zheng, "Bounds on some new weakly singular Wendrofftype integral inequalities and applications," Journal of Inequalities and Applications, vol. 2013, article 159, 2013.

[15] D. Bainov and P. Simeonov, Integral Inequalities and Applications, vol. 57 of Mathematics and Its Applications, Kluwer Academic Publishers, Dordrecht, The Netherlands, 1992.

[16] B. G. Pachpatte, "A note on certain integral inequality," Tamkang Journal of Mathematics, vol. 33, no. 4, pp. 353-358, 2002.

[17] B. G. Pachpatte, "Explicit bounds on Gamidov type integral inequalities," Tamkang Journal of Mathematics, vol. 37, no. 1, pp. $1-9,2006$.

[18] B. G. Pachpatte, "On a certain retarded integral inequality and its applications," Journal of Inequalities in Pure and Applied Mathematics, vol. 5, no. 1, article 19, 2004.

[19] Q.-H. Ma and J. Pečarić, "On certain new nonlinear retarded integral inequalities for functions in two variables and their applications," Journal of the Korean Mathematical Society, vol. 45, no. 1, pp. 121-136, 2008.

[20] F. C. Jiang and F. W. Meng, "Explicit bounds on some new nonlinear integral inequalities with delay," Journal of Computational and Applied Mathematics, vol. 205, no. 1, pp. 479-486, 2007. 


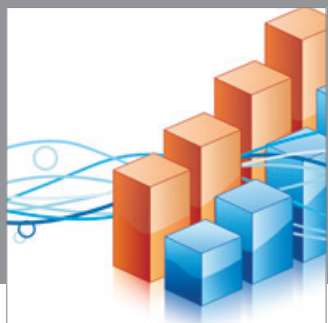

Advances in

Operations Research

mansans

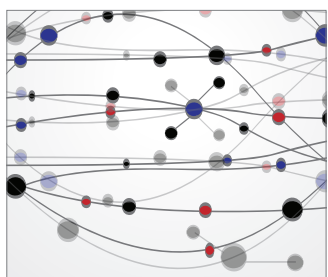

The Scientific World Journal
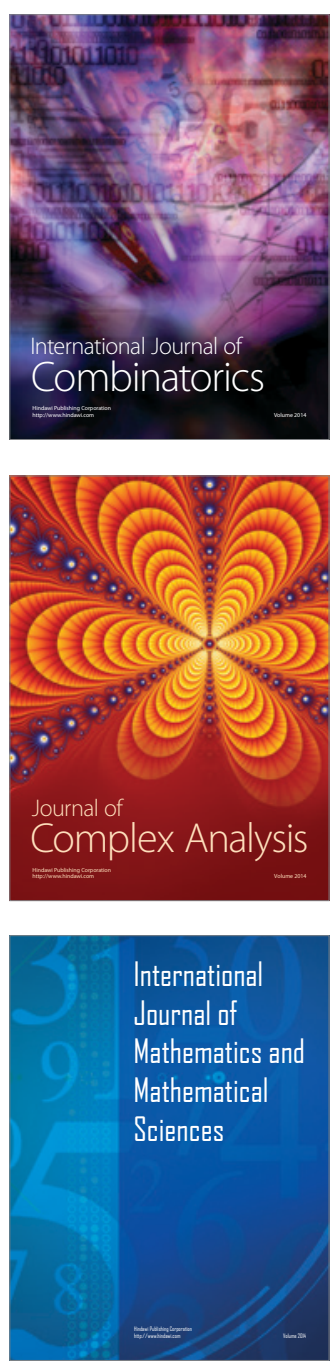
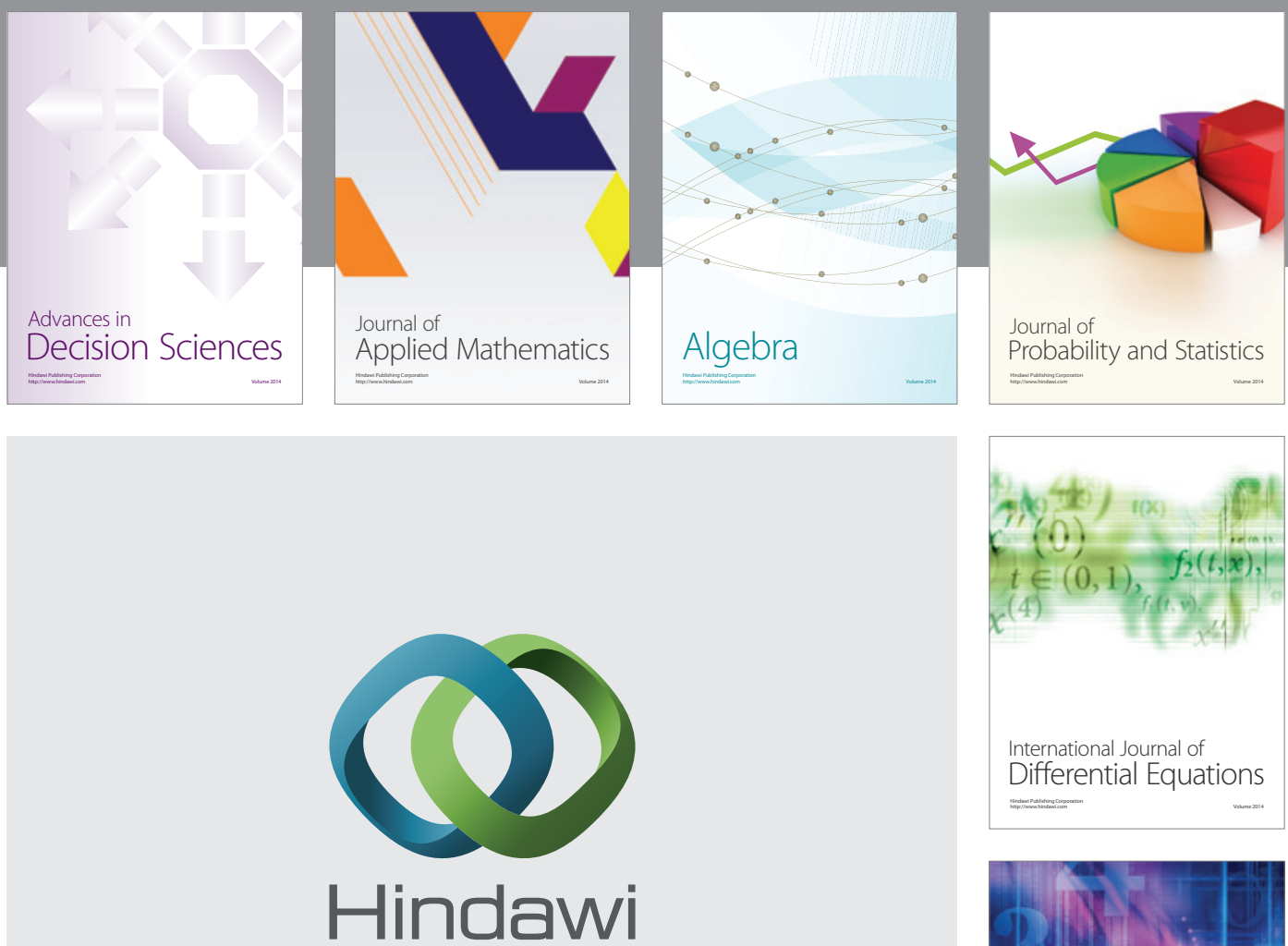

Submit your manuscripts at http://www.hindawi.com
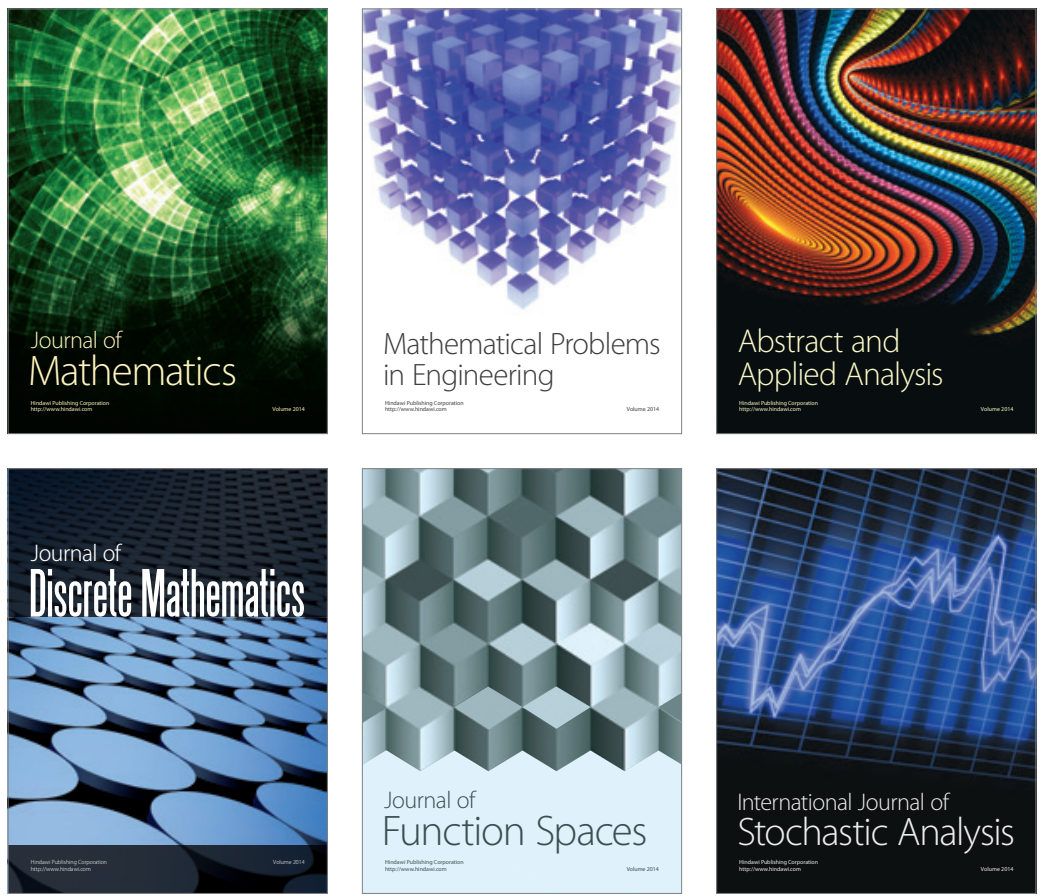

Journal of

Function Spaces

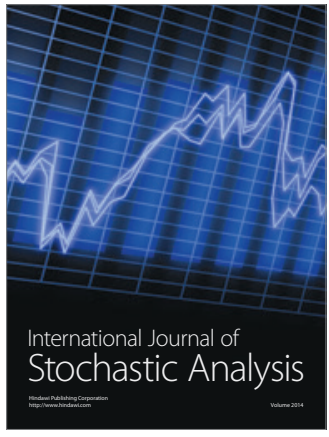

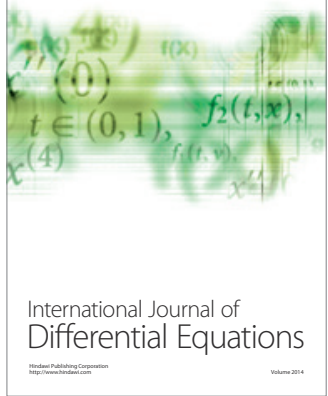
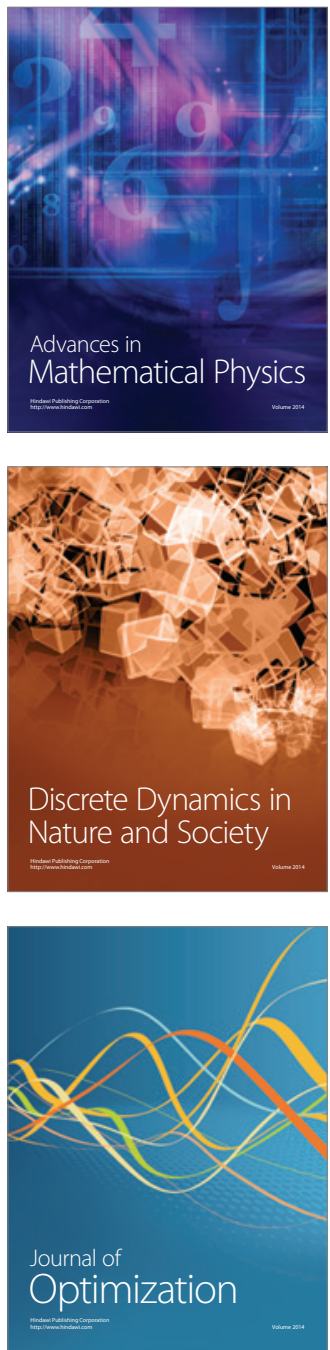